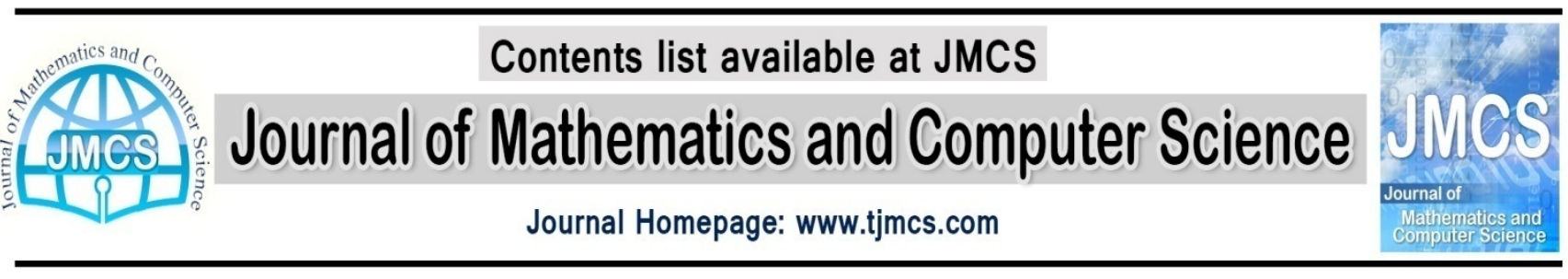

\title{
Prefunctions and System of Differential Equation via Laplace Transform
}

\author{
D. B. Dhaigude \\ Department of Mathematics, Dr. Babasaheb Ambedkar Marathawada UniversityAurangabad, \\ Aurangabad, India
}

S. B. Dhaigude

SPMs Bhivarabai Savant Institute of Technology and Research (for women) Wagholi

Pune-412207, India

Email: suresh.dhaigude@gmail.com

Article history:

Received April 2013

Accepted May 2013

Available online June 2013

\begin{abstract}
The purpose of this paper is to study the system of Linear Differential Equations of first order, Second order and Third order in two variables as well as in three variables and obtained the set of Pre-functions and extended prefunctions are the closed form of solutions via Laplace Transforms technique.
\end{abstract}

Keywords. : Pre-functions, Extended prefunctions, Initial Value Problems and Laplace Transforms

\section{Introduction}

It is well known that some basic functions such as polynomial functions, exponential functions, Logarithmic functions and trigonometric functions have played an important role in development of Mathematical, physical, biological as well as Engineering sciences .Deo and Howell in their paper [1] introduced an alternative method and studied trigonometric and trigonometric like functions. This new approach is simple and useful in the study of differential equations. Khandeparkar, Deo and Dhaigude [3] have defined new kind of Exponential, Trigonometric and hyperbolic functions. They are coined as Pre-functions and extended Pre-functions. It is shown that these pre-functions and extended prefunctions satisfy many interesting properties and relations. Some properties and Laplace Transforms of these functions are studied in the papers [2], [3], [4], [5], [6] and solution of initial value problems for 
non-homogeneous linear ordinary differential equations as well as solutions of voltera integral equations and volltera integro differential equations are obtained.

The aim of this paper is to study the system of Linear Differential Equations of first order, Second order and Third order in two variables and in three variables and obtained the set of Pre-functions and extended prefunctions are the closed form of solutions and Laplace Transforms technique is successfully applied.

\section{Definitions of prefunctions and extended prefunctions}

In this section, we take overview of pre-function and extended pre-functions and Laplace Transforms of these pre-functions [2] and [3] these pre-functions and Laplace Transforms are defined and their properties are also defined. We introduce definitions and Laplace Transforms of some Pre-functions and extended pre-functions.

Definition: The pre-exponential functions is denoted by pexp $(t, \alpha)$ and is defined as,

$$
\operatorname{pexp}(t, \alpha)=1+\sum_{\mathrm{n}=1}^{\infty} \frac{\mathrm{t}^{\mathrm{n}+\alpha}}{\Gamma(\mathrm{n}+1+\alpha)}, \mathrm{t} € \mathrm{R} \text { and } \alpha \geq 0
$$

Also,

$$
\operatorname{pexp}(-t, \alpha)=1+\sum_{\mathrm{n}=1}^{\infty} \frac{(-1)^{\mathrm{n}+\alpha} \mathrm{t}^{\mathrm{n}+\alpha}}{\Gamma(\mathrm{n}+1+\alpha)}, \mathrm{t} € \mathrm{R} \text { and } \alpha \geq 0
$$

The pre-cosine function is denoted by $p \cos (t, \alpha)$ and is defined as,

$$
p \cos (t, \alpha)=1+\sum_{\mathrm{n}=1}^{\infty} \frac{(-1)^{\mathrm{n}} \mathrm{t}^{2 \mathrm{n}+\alpha}}{\Gamma(2 \mathrm{n}+1+\alpha)}, \mathrm{t} € \mathrm{R} \text { and } \alpha \geq 0
$$

The pre-sine function is denoted by $\sin (t, \alpha)$ and is defined as,

$$
p \sin (t, \alpha)=\sum_{\mathrm{n}=0}^{\infty} \frac{(-1)^{\mathrm{n}} \mathrm{t}^{2 \mathrm{n}+\alpha+1}}{\Gamma(2 \mathrm{n}+2+\alpha)}, \mathrm{t} € \mathrm{R} \text { and } \alpha \geq 0
$$

The extended pre function is denoted by $\mathrm{pM}_{32}$

$$
p M 32(t, \alpha)=\sum_{\mathrm{n}=0}^{\infty} \frac{(-1)^{\mathrm{n}} \mathrm{t}^{3 \mathrm{n}+2+\alpha}}{\Gamma(3 \mathrm{n}+3+\alpha)}, \mathrm{t} € \mathrm{R} \text { and } \alpha \geq 0
$$

\section{Laplace Transform of Prefunctions and extended Prefunctions:}

We find the Laplace transforms of $p \exp (t, \alpha)$ : We know that,

$$
\operatorname{pexp}(a t, \alpha)=1+\sum_{\mathrm{n}=1}^{\infty} \frac{(\mathrm{at})^{\mathrm{n}+\alpha}}{\Gamma(\mathrm{n}+1+\alpha)}, \quad \mathrm{t} € \mathrm{R} \quad \text { and } \alpha \geq 0
$$

Now we calculate the Laplace Transforms of this prefunction as follows,

$$
\begin{aligned}
L[p \exp (a t, \alpha)] & =\int_{0}^{\infty} \mathrm{e}^{-\mathrm{st}} p \exp (a t, \alpha) \mathrm{dt} \\
= & \int_{0}^{\infty} \mathrm{e}^{-\mathrm{st}}\left[1+\sum_{\mathrm{n}=1}^{\infty} \frac{(a \mathrm{t})^{\mathrm{n}+\alpha}}{\Gamma(\mathrm{n}+1+\alpha)} \mathrm{dt}\right]
\end{aligned}
$$




$$
\begin{aligned}
& =\int_{0}^{\infty} \mathrm{e}^{-\mathrm{st}} 1 \mathrm{dt}+\sum_{\mathrm{n}=1}^{\infty} \frac{1}{\Gamma(\mathrm{n}+1+\alpha)} \int_{0}^{\infty} \mathrm{e}^{-\mathrm{st}}(\mathrm{at})^{\mathrm{n}+\alpha} \mathrm{dt} \\
& =\frac{1}{s}+\sum_{\mathrm{n}=1}^{\infty} \frac{1}{\Gamma(\mathrm{n}+1+\alpha)} \frac{(\mathrm{a})^{\mathrm{n}+\alpha} \Gamma(\mathrm{n}+1+\alpha)}{\mathrm{s}^{\mathrm{n}+1+\alpha}} \\
& \quad=\frac{1}{s}+\sum_{1}^{\infty} \frac{(\mathrm{a})^{\mathrm{n}+\alpha}}{\mathrm{s}^{\mathrm{n}+1+\alpha}} \\
& =\frac{1}{s}+\frac{(\mathrm{a})^{1+\alpha}}{(\mathrm{s}-\mathrm{a}) \mathrm{s}^{1+\alpha}}
\end{aligned}
$$

In particular, for $a=1$,

$$
L[p \exp (t, \alpha)]=\frac{1}{s}+\frac{1}{(\mathrm{~s}-1) \mathrm{s}^{1+\alpha}}
$$

In particular $\alpha=0$,

$$
L[p \exp (t, 0)]=L[p \exp (t)]=\frac{1}{(s-1)}
$$

Clearly,

$$
L^{-1}\left[\frac{1}{s}+\frac{(\mathrm{a})^{1+\alpha}}{(\mathrm{s}-\mathrm{a}) \mathrm{s}^{1+\alpha}}\right]=\operatorname{pexp}(a t, \alpha)
$$

Laplace transforms of other prefunctions can be derived and listed as follows,

1. $L[p \exp (-a t, \alpha)]=\frac{1}{s}-\frac{(-1)^{\alpha}(a)^{1+\alpha}}{(s+a) s^{1+\alpha}}$

2. $L[p \sin (a t, \alpha)]=\frac{(\mathrm{a})^{1+\alpha}}{\left(\mathrm{s}^{2}+\mathrm{a}^{2}\right) \mathrm{s}^{\alpha}}$

3. $L[p \cos (a t, \alpha)]=\frac{1}{s}-\frac{\mathrm{a}^{\alpha+2}}{\left(\mathrm{~s}^{2}+\mathrm{a}^{2}\right) \mathrm{s}^{1+\alpha}}$

4. $L[p \sinh (a t, \alpha)]=\frac{(\mathrm{a})^{1+\alpha}}{\left(\mathrm{s}^{2}-\mathrm{a}^{2}\right) \mathrm{s}^{\alpha}}$

5. $L[p \cosh (a t, \alpha)]=\frac{1}{s}+\frac{\mathrm{a}^{\alpha+2}}{\left(\mathrm{~s}^{2}-\mathrm{a}^{2}\right) \mathrm{s}^{1+\alpha}}$

6. $L\left[p M_{30}(a t, \alpha)\right]=\frac{1}{\mathrm{~s}}-\frac{(\mathrm{a})^{3+\alpha}}{\left(\mathrm{s}^{3}+\mathrm{a}^{3}\right) \mathrm{s}^{\alpha+1}}$

7. $L\left[p M_{31}(a t, \alpha)\right]=\frac{(a)^{1+\alpha}}{\left(s^{3}+a^{3}\right) s^{\alpha-1}}$

8. $L\left[p M_{32}(a t, \alpha)\right]=\frac{(\mathrm{a})^{2+\alpha}}{\left(\mathrm{s}^{3}+\mathrm{a}^{3}\right) \mathrm{s}^{\alpha}}$

9. $L\left[p N_{30}(a t, \alpha)\right]=\frac{1}{\mathrm{~s}}+\frac{(\mathrm{a})^{3+\alpha}}{\left(\mathrm{s}^{3}-\mathrm{a}^{3}\right) \mathrm{s}^{\alpha+1}}$

10. $L\left[p N_{31}(a t, \alpha)\right]=\frac{(a)^{1+\alpha}}{\left(s^{3}-a^{3}\right) s^{\alpha-1}}$ 
11. $L\left[p N_{32}(a t, \alpha)\right]=\frac{(\mathrm{a})^{2+\alpha}}{\left(\mathrm{s}^{3}-\mathrm{a}^{3}\right) \mathrm{s}^{\alpha}}$

Note that on putting $\alpha=0$ and $a=1$ above we get Laplace Transforms of exponential functions, trigonometric functions and hyperbolic function.

\section{Initial Value Problems for System of Differential Equations:}

The solution of simultaneous differential equations with initial conditions can be obtained by applying Laplace Transform method. First we discuss the solution of first order system of ordinary differential equations with suitable initial conditions.

\section{First Order System of Differential Equations:}

IVP-I. Consider the system of first order differential equations,

$$
\begin{aligned}
& x^{\prime}(t, \alpha)+y(t, \alpha)=0 \\
& y^{\prime}(t, \alpha)-x(t, \alpha)=\frac{t^{\alpha}}{\Gamma(\alpha+1)}-1
\end{aligned}
$$

With the initial conditions, $x(0, \alpha)=1, y(0, \alpha)=0$

Apply Laplace transform to both the sides of differential equations (1.1) and (1.2), we have,

$$
\begin{gathered}
\qquad\left\{x^{\prime}(t, \alpha)\right\}+L\{y(t, \alpha)\}=0 \\
L\left\{y^{\prime}(t, \alpha)\right\}-L\{x(t, \alpha)\}=L\left\{\frac{t^{\alpha}}{\Gamma(\alpha+1)}\right\}-L\{1\} \\
s L\{x(t, \alpha)\}-x(0, \alpha)+L\{y(t, \alpha)\}=0 \\
\text { And } \quad s L\{y(t, \alpha)\}-y(0, \alpha)-L\{x(t, \alpha)\}=\frac{1}{\Gamma(\alpha+1)} \frac{\Gamma(\alpha+1)}{s^{\alpha+1}}-\frac{1}{s}
\end{gathered}
$$

Consider, $L\{x(t, \alpha)=X(s, \alpha), L\{y(t, \alpha)=Y(s, \alpha)$. Using initial conditions (1.3), we obtain the System of equations in $X$ and $Y$

$$
\begin{gathered}
s X(s, \alpha)+Y(s, \alpha)=1 \\
-X(s, \alpha)+s Y(s, \alpha)=\frac{1}{s^{\alpha+1}}-\frac{1}{s}
\end{gathered}
$$

Multiply equation (1.5) by s and add with equation (1.4), we get,

$$
\begin{aligned}
\left(s^{2}+1\right) Y(s, \alpha) & =\frac{1}{s^{\alpha}} \\
Y(s, \alpha) & =\frac{1}{\left(s^{2}+1\right) s^{\alpha}}
\end{aligned}
$$

Taking inverse Laplace transform of both sides, we have, 


$$
\begin{gathered}
L^{-1}\{Y(s, \alpha)\}=L^{-1}\left\{\frac{1}{\left(s^{2}+1\right) s^{\alpha}}\right\} \\
y(t, \alpha)=p \sin (t, \alpha)
\end{gathered}
$$

Now we obtain prefunction $x(t, \alpha)$, multiply equation (1.4) by $\mathrm{s}$ and subtract equation (1.5) from it, we have,

$$
\begin{aligned}
\left(s^{2}+1\right) X(s, \alpha) & =\frac{s^{2}+1}{s}-\frac{1}{s^{\alpha+1}} \\
X(s, \alpha) & =\frac{1}{s}-\frac{1}{\left(s^{2}+1\right) s^{\alpha+1}}
\end{aligned}
$$

Taking inverse Laplace transform of both sides, we have,

$$
\begin{aligned}
L^{-1}\{X(s, \alpha)\} & =L^{-1}\left\{\frac{1}{s}-\frac{1}{\left(s^{2}+1\right) s^{\alpha+1}}\right\} \\
x(t, \alpha) & =p \cos (t, \alpha)
\end{aligned}
$$

The solution set of the IVP (1.1)-(1.3) is $\{x(t, \alpha)=p \cos (t, \alpha), y(t, \alpha)=p \sin (t, \alpha)\}$.

IVP-II. Consider the system of first order differential equations,

$$
\begin{aligned}
& x^{\prime}(t, \alpha)+z(t, \alpha)=0 \\
& y^{\prime}(t, \alpha)-x(t, \alpha)=\frac{t^{\alpha}}{\Gamma(\alpha+1)}-1 \\
& z^{\prime}(t, \alpha)-y(t, \alpha)=0
\end{aligned}
$$

With the initial conditions,

$$
x(0, \alpha)=1, y(0, \alpha)=0, z(0, \alpha)=0
$$

Apply Laplace transform to both the sides of differential equations (2.1), (2.2), and (2.3) we have,

$$
\begin{aligned}
& L\left\{x^{\prime}(t, \alpha)\right\}+L\{z(t, \alpha)\}=0 \\
& L\left\{y^{\prime}(t, \alpha)\right\}-L\{x(t, \alpha)\}=L\left\{\frac{t^{\alpha}}{\Gamma(\alpha+1)}\right\}-L\{1\} \\
& L\left\{z^{\prime}(t, \alpha)\right\}-L\{y(t, \alpha)\}=0
\end{aligned}
$$

Simplification it gives,

And

$$
\begin{aligned}
& s L\{x(t, \alpha)\}-x(0, \alpha)+L\{z(t, \alpha)\}=0 \\
& s L\{y(t, \alpha)\}-y(0, \alpha)-L\{x(t, \alpha)\}=\frac{1}{\Gamma(\alpha+1)} \frac{\Gamma(\alpha+1)}{s^{\alpha+1}}-\frac{1}{s} \\
& s L\{z(t, \alpha)\}-z(0, \alpha)-L\{y(t, \alpha)\}=0
\end{aligned}
$$


Suppose,

$$
L\{x(t, \alpha)\}=X(s, \alpha), L\{y(t, \alpha)\}=Y(s, \alpha), L\{z(t, \alpha)\}=Z(s, \alpha)
$$

Using initial condition (2.4), we get the system of equations in $X, Y$ and $Z$

$$
\begin{aligned}
& s X(s, \alpha)+Z(s, \alpha)=1 \\
& -X(s, \alpha)+s Y(s, \alpha)=\frac{1}{s^{\alpha+1}}-\frac{1}{s} \\
& -Y(s, \alpha)+s Z(s, \alpha)=0
\end{aligned}
$$

We write this system of equations in matrix form as,

$$
\left[\begin{array}{ccc}
S & 0 & 1 \\
-1 & S & 0 \\
0 & -1 & s
\end{array}\right]\left[\begin{array}{l}
X \\
Y \\
Z
\end{array}\right]=\left[\begin{array}{c}
1 \\
\frac{1}{s^{\alpha+1}}-\frac{1}{s} \\
0
\end{array}\right]
$$

We know by matrix theory,

$$
X=\frac{D_{x}}{D}, \quad Y=\frac{D_{y}}{D}, Z=\frac{D_{z}}{D}
$$

Where,

$$
\begin{gathered}
D=\left|\begin{array}{ccc}
s & 0 & 1 \\
-1 & s & 0 \\
0 & -1 & s
\end{array}\right|=s^{3}+1 \\
D_{x}=\left|\begin{array}{cccc}
1 & 0 & -1 \\
\frac{1}{s^{\alpha+1}}-\frac{1}{s} & s & 0 \\
0 & -1 & s
\end{array}\right|=s^{2}+\frac{1}{s}-\frac{1}{s^{\alpha+1}} \\
D_{y}=\left|\begin{array}{ccc}
s & 1 & 1 \\
-1 & \frac{1}{s^{\alpha+1}}-\frac{1}{s} & 0 \\
0 & 0 & s
\end{array}\right|=\frac{1}{s^{\alpha-1}} \\
D_{z}=\left|\begin{array}{ccc}
s & 0 & 1 \\
-1 & s & \frac{1}{s^{\alpha+1}}-\frac{1}{s} \\
0 & -1 & 0
\end{array}\right|=\frac{1}{s^{\alpha}}
\end{gathered}
$$

Substituting these values in equation (2.5), then we obtain

$$
\begin{gathered}
X(s, \alpha)=\frac{1}{s}-\frac{1}{\left(s^{3}+1\right) s^{\alpha+1}} \\
Y(s, \alpha)=\frac{1}{\left(s^{3}+1\right) s^{\alpha-1}} \\
Z(s, \alpha)=\frac{1}{\left(s^{3}+1\right) s^{\alpha}}
\end{gathered}
$$


In each case, taking inverse Laplace transform of both sides, we have,

$$
\begin{gathered}
\mathrm{x}(t, \alpha)=L^{-1}\left\{\frac{1}{s}-\frac{1}{\left(s^{3}+1\right) s^{\alpha+1}}\right\}=p M_{30}(t, \alpha) \\
\mathrm{y}(t, \alpha)=L^{-1}\left\{\frac{1}{\left(s^{3}+1\right) s^{\alpha-1}}\right\}=p M_{31}(t, \alpha) \\
\mathrm{z}(t, \alpha)=L^{-1}\left\{\frac{1}{\left(s^{3}+1\right) s^{\alpha}}\right\}=p M_{32}(t, \alpha)
\end{gathered}
$$

The solution set of the IVP (2.1)-(2.4) is,

$$
\left\{\mathrm{x}(t, \alpha)=p M_{30}(t, \alpha), \mathrm{y}(t, \alpha)=p M_{31}(t, \alpha), \mathrm{z}(t, \alpha)=p M_{32}(t, \alpha)\right\}
$$

\section{Second Order System of Differential Equation:}

IVP-III: Consider the system of first order differential equations,

$$
\begin{gathered}
x^{\prime \prime}(t, \alpha)-y^{\prime}(t, \alpha)=0 \\
y^{\prime \prime}(t, \alpha)-x^{\prime}(t, \alpha)=\frac{t^{\alpha-1}}{\Gamma(\alpha)}
\end{gathered}
$$

With initial conditions,

$$
x(0, \alpha)=1, y(0, \alpha)=0, x^{\prime}(0, \alpha)=0, y^{\prime}(0, \alpha)=0
$$

Apply Laplace transform to both the sides of differential equations (3.1) and (3.2),

We have,

$$
\begin{gathered}
L\left\{x^{\prime \prime}(t, \alpha)\right\}-L\left\{y^{\prime}(t, \alpha)\right\}=0 \\
L\left\{y^{\prime \prime}(t, \alpha)\right\}-L\left\{x^{\prime}(t, \alpha)\right\}=L\left\{\frac{t^{\alpha-1}}{\Gamma(\alpha)}\right\} \\
s^{2} L\{x(t, \alpha)\}-s x(0, \alpha)-x^{\prime}(0, \alpha)-s L\{y(t, \alpha)\}+y(t, \alpha)=0
\end{gathered}
$$

And $s^{2} L\{y(t, \alpha)\}-s y(0, \alpha)-y^{\prime}(0, \alpha)-L\{x(t, \alpha)\}+x(0, \alpha)=\frac{1}{\Gamma(\alpha)} \frac{\Gamma(\alpha)}{s^{\alpha}}$

Consider, $L\{x(t, \alpha)\}=X(s, \alpha), L\{y(t, \alpha)\}=Y(s, \alpha)$, using initial condition (3.3)

We get the system of equations in $X, Y$

$$
\begin{aligned}
& s^{2} X(s, \alpha)-s Y(s, \alpha)=s \\
& -s X(s, \alpha)+s^{2} Y(s, \alpha)=\frac{1}{s^{\alpha}}-1
\end{aligned}
$$

Multiply equation (3.5) by s and add with equation (3.4), we obtain 


$$
\begin{aligned}
& \left(s^{3}-s\right) Y(s, \alpha)=\frac{1}{s^{\alpha-1}} \\
& Y(s, \alpha)=\frac{1}{\left(s^{2}-1\right) s^{\alpha}}
\end{aligned}
$$

Taking inverse Laplace transform of both sides, we have,

$$
y(t, \alpha)=L^{-1}\left\{\frac{1}{\left(s^{2}-1\right) s^{\alpha}}\right\}=p \sinh (t, \alpha)
$$

Also multiply equation (3.4) by s and add with equation (3.5), we obtain

$$
\begin{aligned}
\left(s^{3}-s\right) X(s, \alpha) & =\left(s^{2}-1\right)+\frac{1}{s^{\alpha}} \\
X(s, \alpha) & =\frac{1}{s}+\frac{1}{\left(s^{2}-1\right) s^{\alpha+1}}
\end{aligned}
$$

Taking inverse Laplace transform of both sides, we have,

$$
\mathrm{x}(t, \alpha)=L^{-1}\left\{\frac{1}{s}+\frac{1}{\left(s^{2}-1\right) s^{\alpha+1}}\right\}=p \cosh (t, \alpha)
$$

The solution set of the IVP (3.1)-(3.3) is $\{x(t, \alpha)\}=p \cosh (t, \alpha), y(t, \alpha)=p \sinh (t, \alpha)\}$

IVP-IV: Consider the system of second order differential equations

$$
\begin{aligned}
& x^{\prime \prime}(t, \alpha)-z^{\prime}(t, \alpha)=0 \\
& y^{\prime \prime}(t, \alpha)-x^{\prime}(t, \alpha)=\frac{t^{\alpha-1}}{\Gamma(\alpha)} \\
& z^{\prime \prime}(t, \alpha)-y^{\prime}(t, \alpha)=0
\end{aligned}
$$

With the initial conditions,

$$
x(0, \alpha)=1, y(0, \alpha)=z(0, \alpha)=x^{\prime}(0, \alpha)=y^{\prime}(0, \alpha)=z^{\prime}(0, \alpha)=0
$$

Apply Laplace transform to both the sides of differential equations (4.1), (4.2) and (4.3), we have,

$$
\begin{aligned}
& L\left\{x^{\prime \prime}(t, \alpha)\right\}-L\left\{z^{\prime}(t, \alpha)\right\}=0 \\
& L\left\{y^{\prime \prime}(t, \alpha)\right\}-L\left\{x^{\prime}(t, \alpha)\right\}=L\left\{\frac{t^{\alpha-1}}{\Gamma(\alpha)}\right\} \\
& L\left\{z^{\prime \prime}(t, \alpha)\right\}-L\left\{y^{\prime}(t, \alpha)\right\}=0
\end{aligned}
$$

Suppose,

$$
L\{x(t, \alpha)\}=X(s, \alpha), L\{y(t, \alpha)\}=Y(s, \alpha), L\{z(t, \alpha)\}=Z(s, \alpha)
$$

Using initial condition (4.4), we get the system of equations in $\mathrm{X}, \mathrm{Y}$ and $\mathrm{Z}$

$$
s^{2} X(s, \alpha)-s Z(s, \alpha)=s
$$




$$
\begin{gathered}
-s X(s, \alpha)+s^{2} Y(s, \alpha)=\frac{1}{s^{\alpha}}-1 \\
-Y(s, \alpha)+s^{2} Z(s, \alpha)=0
\end{gathered}
$$

We write this system of equations in matrix form as,

$$
\left[\begin{array}{ccc}
s^{2} & 0 & -s \\
-s & s^{2} & 0 \\
0 & -s & s^{2}
\end{array}\right]\left[\begin{array}{l}
X \\
Y \\
Z
\end{array}\right]=\left[\begin{array}{c}
s \\
\frac{1}{s^{\alpha}}-1 \\
0
\end{array}\right]
$$

We know by matrix theory, $\quad X=\frac{D_{x}}{D}, Y=\frac{D_{y}}{D}, Z=\frac{D_{z}}{D}$

Where,

$$
\begin{gathered}
D=\left|\begin{array}{ccc}
s^{2} & 0 & -s \\
-s & s^{2} & 0 \\
0 & -s & s^{2}
\end{array}\right|=s^{3}\left(s^{3}-1\right) \\
D_{x}=\left|\begin{array}{ccc}
s & 0 & -s \\
\frac{1}{s^{\alpha}}-1 & s^{2} & 0 \\
0 & -s & s^{2}
\end{array}\right|=s^{5}-s^{2}+\frac{s^{2}}{s^{\alpha}} \\
D_{y}=\left|\begin{array}{ccc}
s^{2} & s & -s \\
-s & \frac{1}{s^{\alpha}}-1 & 0 \\
0 & 0 & s^{2}
\end{array}\right|=\frac{s^{4}}{s^{\alpha}} \\
D_{z}=\left|\begin{array}{ccc}
s^{2} & 0 & s \\
-s & s^{2} & \frac{1}{s^{\alpha}}-1 \\
0 & -s & 0
\end{array}\right|=\frac{s^{3}}{s^{\alpha}}
\end{gathered}
$$

Substituting these values in equation (4.5), then we obtain

$$
\begin{gathered}
X(s, \alpha)=\frac{1}{s}+\frac{1}{\left(s^{3}-1\right) s^{\alpha+1}} \\
Y(s, \alpha)=\frac{1}{\left(s^{3}-1\right) s^{\alpha-1}} \\
Z(s, \alpha)=\frac{1}{\left(s^{3}-1\right) s^{\alpha}}
\end{gathered}
$$

In each case, taking inverse Laplace transform of both sides, we have,

$$
\begin{gathered}
\mathrm{x}(t, \alpha)=L^{-1}\left\{\frac{1}{s}+\frac{1}{\left(s^{3}-1\right) s^{\alpha+1}}\right\}=p N_{30}(t, \alpha) \\
\mathrm{y}(t, \alpha)=L^{-1}\left\{\frac{1}{\left(s^{3}-1\right) s^{\alpha-1}}\right\}=p N_{31}(t, \alpha) \\
\mathrm{z}(t, \alpha)=L^{-1}\left\{\frac{1}{\left(s^{3}-1\right) s^{\alpha}}\right\}=p N_{32}(t, \alpha)
\end{gathered}
$$


The solution set of the IVP (4.1)-(4.4) is,

$$
\left\{\mathrm{x}(t, \alpha)=p N_{30}(t, \alpha), \mathrm{y}(t, \alpha)=p N_{31}(t, \alpha), \mathrm{z}(t, \alpha)=p N_{32}(t, \alpha)\right\}
$$

\section{Third Order System of Differential Equation:}

IVP-V: Consider the system of second order differential equations,

$$
\begin{aligned}
& \mathrm{x}^{\prime \prime \prime}(t, \alpha)+z^{\prime \prime}(t, \alpha)=0 \\
& y^{\prime \prime \prime}(t, \alpha)-x^{\prime \prime}(t, \alpha)=\frac{t^{\alpha-2}}{\Gamma(\alpha-1)} \\
& z^{\prime \prime \prime}(t, \alpha)-y^{\prime \prime}(t, \alpha)=0
\end{aligned}
$$

with the initial conditions,

$$
\begin{aligned}
& x(0, \alpha)=1, y(0, \alpha)=z(0, \alpha)=x^{\prime}(0, \alpha)=y^{\prime}(0, \alpha)=z^{\prime}(0, \alpha)=0 \\
& x^{\prime \prime}(0, \alpha)=y^{\prime \prime}(0, \alpha)=z^{\prime \prime}(0, \alpha)=0
\end{aligned}
$$

Apply Laplace transform to both the sides of differential equations (5.1),(5.2) and (5.3), we have,

$$
\begin{gathered}
L\left\{x^{\prime \prime \prime}(t, \alpha)\right\}+L\left\{z^{\prime \prime}(t, \alpha)\right\}=0 \\
L\left\{y^{\prime \prime \prime}(t, \alpha)\right\}-L\left\{x^{\prime \prime}(t, \alpha)\right\}=L\left\{\frac{t^{\alpha-2}}{\Gamma(\alpha-1)}\right\} \\
L\left\{z^{\prime \prime \prime}(t, \alpha)\right\}-L\left\{y^{\prime \prime}(t, \alpha)\right\}=0
\end{gathered}
$$

Suppose, $\quad L\{x(t, \alpha)\}=X(s, \alpha), L\{y(t, \alpha)\}=Y(s, \alpha), L\{z(t, \alpha)\}=Z(s, \alpha)$

Using initial condition (5.4), we get the system of equations in $\mathrm{X}, \mathrm{Y}$ and $\mathrm{Z}$

$$
\begin{aligned}
s^{3} X(s, \alpha)+s^{2} Z(s, \alpha) & =s^{2} \\
-s^{2} X(s, \alpha)+s^{3} Y(s, \alpha) & =\frac{1}{s^{\alpha-1}}-s \\
s^{3} Z(s, \alpha)-s^{2} Y(s, \alpha) & =0
\end{aligned}
$$

we write this system of equations in matrix form as,

$$
\left[\begin{array}{ccc}
s^{3} & 0 & s^{2} \\
-s^{2} & s^{3} & 0 \\
0 & -s^{2} & s^{3}
\end{array}\right]\left[\begin{array}{l}
X \\
Y \\
Z
\end{array}\right]=\left[\begin{array}{c}
s^{2} \\
\frac{1}{s^{\alpha-1}}-s \\
0
\end{array}\right]
$$

we know by matrix theory,

$$
X=\frac{D_{x}}{D}, \quad Y=\frac{D_{y}}{D}, Z=\frac{D_{z}}{D}
$$

where, 


$$
\begin{aligned}
D & =\left|\begin{array}{ccc}
s^{3} & 0 & s^{2} \\
-s^{2} & s^{3} & 0 \\
0 & -s^{2} & s^{3}
\end{array}\right|=s^{6}\left(s^{3}+1\right) \\
D_{x} & =\left|\begin{array}{ccc}
s^{2} & 0 & s^{2} \\
\frac{1}{s^{\alpha-1}}-s & s^{3} & 0 \\
0 & -s^{2} & s^{3}
\end{array}\right|=s^{8}+s^{5}-\frac{1}{s^{\alpha-5}} \\
D_{y} & =\left|\begin{array}{ccc}
s^{3} & s^{2} & s^{2} \\
-s^{2} & \frac{1}{s^{\alpha-1}}-s & 0 \\
0 & 0 & s^{3}
\end{array}\right|=\frac{s^{6}}{s^{\alpha-1}} \\
D_{z} & =\left|\begin{array}{ccc}
s^{3} & 0 & s^{2} \\
-s^{2} & s^{3} & \frac{1}{s^{\alpha-1}}-s \\
0 & -s^{2} & 0
\end{array}\right|=\frac{s^{3}}{s^{\alpha-1}}
\end{aligned}
$$

Substituting these values in equation (5.5), then we obtain

$$
\begin{aligned}
& X(s, \alpha)=\frac{1}{s}-\frac{1}{\left(s^{3}+1\right) s^{\alpha+1}} \\
& Y(s, \alpha)=\frac{1}{\left(s^{3}+1\right) s^{\alpha-1}} \\
& Z(s, \alpha)=\frac{1}{\left(s^{3}+1\right) s^{\alpha}}
\end{aligned}
$$

In each case, taking inverse Laplace transform of both sides, we have,

$$
\begin{gathered}
\mathrm{x}(t, \alpha)=L^{-1}\left\{\frac{1}{s}-\frac{1}{\left(s^{3}+1\right) s^{\alpha+1}}\right\}=p M_{30}(t, \alpha) \\
\mathrm{y}(t, \alpha)=L^{-1}\left\{\frac{1}{\left(s^{3}+1\right) s^{\alpha-1}}\right\}=p M_{31}(t, \alpha) \\
\mathrm{z}(t, \alpha)=L^{-1}\left\{\frac{1}{\left(s^{3}+1\right) s^{\alpha}}\right\}=p M_{32}(t, \alpha)
\end{gathered}
$$

The solution set of the IVP (5.1)-(5.4) is,

$$
\left\{\mathrm{x}(t, \alpha)=p M_{30}(t, \alpha), \mathrm{y}(t, \alpha)=p M_{31}(t, \alpha), \mathrm{z}(t, \alpha)=p M_{32}(t, \alpha)\right\}
$$

Conclusion: In this paper we have studied the system of Linear Differential Equations of first Order, Second order and Third order in two variables as well as in three variables and the set of Prefunctions and extended Prefunctions are the closed form of solutions, Laplace Transforms method is successfully applied.

Acknowledgements: The second authors is thankful to Principal Dr.D.M.Yadav, JSPMs Bhivarabai Savant Institute of Technology and Research (for women) Wagholi, Pune for the given the time and support to this work. 


\section{References:}

[1] S.G.Deo and G.W.Howell: A Highway to Trigonometry, Bull. Marathawada Mathematical society.1 (2000), 26-62

[2] R.B.Khandeparkar, S.G.Deo and D.B.Dhaigude: Preexponential and Pre trigonometric Functions, Comm. Appl.Anal.14 (2010), no.1, 99-116

[3] R.B.Khandeparkar: Pretrigonometric and Prehyperbolic Functions via Laplace Transform, Neural, Parallel, and Scientific Computations, 18 (2010), 423-432.

[4] S.B.Dhaigude and Chandradeepa D Dhaigude: Some Results on Pre-functions and Differential Equations, Bull. Marathawada Mathematical society. 12 No .2 (2011), 18-23.

[5] S.B.Dhaigude and Chandradeepa D Dhaigude: Prefunctions and Integral Equations via Laplace Transforms, International Journal of Engineering Sciences, 2(5) may 2013, 204-209.

[6] Mustafa Mahmoudi, Mohmmad V.Kazemi: Solving Singular BVPs for Ordinary Differential Equations by modified Homotopy perturbation method, the journal of mathematics and computer science, Vol.7, Issue 2,138-143. (2013)

[7] M.Rabbani, B Zarali: Solution of Fredholm Integro- Differential Equations System by modified decomposition method, The journal of mathematics and computer science, Vol.5, Issue 4,258-264. (2012).

[8] Srivastava R.S.L., Engineering Mathematics, Vol.1 and 2, McGraw Hill, 1980.

[9] R.B.Khandeparkar; Studies in Matrix Functions and Differential Equations, Ph.D.Thesis, Dr.Babasaheb Ambedkar Marathawada University Aurangabad.sept.2010 India. 